\title{
Analgesia Preemptiva com S(+)Cetamina e Bupivacaína Peridural em Histerectomia Abdominal *
}

\section{Preemptive Analgesia with Epidural Bupivacaine and S(+)Ketamine in Abdominal Hysterectomy}

Ferdinand Edson de Castro ${ }^{1}$; João Batista Santos Garcia, TSA ${ }^{2}$

\section{RESUMO}

Castro FE, Garcia JBS - Analgesia Preemptiva com S(+)Cetamina e Bupivacaína Peridural em Histerectomia Abdominal

\section{JUSTIFICATIVA E OBJETIVOS: O presente estudo investiga a} capacidade de o antagonista do receptor NMDA, $S(+)$ cetamina, associado à injeção peridural de anestésico local (bupivacaína), previamente administrado à incisão promover analgesia preemptiva em pacientes submetidas a histerectomia total abdominal.

MÉTODO: Foram avaliadas 30 pacientes, distribuídas aleatoriamente em dois grupos de igual tamanho e estudadas prospectivamente de forma encoberta. Injeção peridural e inserção de cateter foram realizadas entre os interespaços de $L_{1}-L_{2}$. No grupo I (G1, $\left.n=15\right)$, as pacientes receberam, por via peridural, $17 \mathrm{~mL}$ de bupivacaína a 0,25\%, sem vasoconstritor, associados a $30 \mathrm{mg}$ de $\mathrm{S}(+)$ cetamina $(3 \mathrm{~mL}$ ), trinta minutos antes da incisão cirúrgica; após 30 minutos da incisão, receberam $20 \mathrm{~mL}$ de solução fisiológica a $0,9 \%$. No grupo $2(\mathrm{G} 2, n=15)$, receberam $20 \mathrm{~mL}$ de solução fisiológica, por via peridural, 30 minutos antes da incisão, sendo feita administração de $17 \mathrm{~mL}$ de bupivacaína a $0,25 \%$ associados a $30 \mathrm{mg}$ de $S(+)$ cetamina (3 $\mathrm{mL})$, trinta minutos depois da incisão. Após a injeção peridural, realizou-se anestesia geral com propofol, pancurônio, $\mathrm{O}_{2}$ e isoflurano. Para analgesia pós-operatória foi usada solução peridural em bolus de fentanil associada à bupivacaína, em intervalo mínimo de quatro horas e suplementação com dipirona, se necessária. Avaliou-se a intensidade da dor através de escala numérica e verbal (ao despertar, 6, 12, 18 e 24 horas após o término da operação), o tempo necessário para solicitar pela primeira vez o analgésico e o consumo total de analgésicos.

RESULTADOS: Não houve diferença significativa entre os grupos em relação ao tempo para solicitar analgésicos pela primeira vez, ao consumo de analgésicos e aos escores de dor pelas escalas numérica e verbal.
CONCLUSÕES: Não foi possivel demonstrar efeito preemptivo com a utilização peridural de $S(+)$ cetamina e bupivacaína nas doses utilizadas em histerectomia abdominal.

Unitermos: ANALGESIA: peridural, preemptiva; ANESTÉSICOS: bupivacaína, cetamina.

\section{SUMMARY}

Castro FE, Garcia JBS - Preemptive Analgesia with Epidural Bupivacaine and $\mathrm{S}(+)$ Ketamine in Abdominal Hysterectomy

BACKGROUND AND OBJECTIVES: This study investigates the ability of epidural $S(+)$ ketamine, NMDA receptor antagonist, plus local anesthetic (bupivacaine) injection to promote preemptive analgesia in patients undergoing total abdominal hysterectomy, when this solution is administered before surgical incision.

METHODS: Participated in this prospective double-blind study 30 patients were randomly assigned in two equal groups. Epidural injection and catheter insertion were performed at $L_{1}-L_{2}$ interspace. Group 1 (G1) patients received $17 \mathrm{~mL}$ bupivacaine $0.25 \%$ plus $3 \mathrm{~mL} \mathrm{~S}(+)$ ketamine $(30 \mathrm{mg}), 30 \mathrm{~min}$ before surgical incision, followed by $20 \mathrm{~mL}$ saline $30 \mathrm{~min}$ after incision. Group 2 (G2) patients received $20 \mathrm{~mL}$ saline $30 \mathrm{~min}$ before surgical incision, followed by $17 \mathrm{~mL}$ bupivacaine $0.25 \%$ plus 3 $\mathrm{mL} \mathrm{S}(+)$ ketamine $(30 \mathrm{mg}) 30 \mathrm{~min}$ after incision. General anesthesia was induced with propofol, pancuronium, $\mathrm{O}_{2}$ and isoflurane. Postoperative analgesia consisted of epidural fentanyl plus bupivacaine bolus with 4 h minimal interval. If necessary, IV dipyrone supplementation was administered. Patients were evaluated for analgesia by a verbal and numeric scale (at recovery and every six hours until 24 postoperative hours). Time to first analgesic request and total analgesic requirements were recorded.

RESULTS: There were no significant differences between groups in time to first analgesic request, total analgesic consumption and numeric or verbal scale pain scores.

CONCLUSIONS: This study failed to demonstrate a preemptive effect of epidural administration of bupivacaine and $S(+)$ ketamine in the doses tested for abdominal hysterectomy.

\footnotetext{
${ }^{*}$ Recebido do (Received from) Hospital Universitário Materno-Infantil da Universidade Federal do Maranhão (HU-UFMA), São Luís, MA

1. Professor Adjunto da Disciplina de Fisiologia da Universidade Federal do Maranhão; Anestesiologista Assistente do HU-UFMA

2. Professor Adjunto Doutor da Disciplina de Anestesiologia da Universidade Federal do Maranhão. Responsável pelo Serviço de Dor do Hospital Universitário (HU-UFMA)
}

Apresentado (Submitted) em 05 de julho de 2004 Aceito (Accepted) para publicação em 27 de outubro de 2004

Endereço para correspondência (Correspondence to) Dr. João Batista Santos Garcia

Av. dos Holandeses, 213/701 Ponta D'Areia

65085-450 São Luís, MA

E-mail: jbgarcia@uol.com.br

(C) Sociedade Brasileira de Anestesiologia, 2005
Key Words: ANALGESIA, epidural, preemptive; ANESTHETICS: bupivacaine, ketamine

\section{INTRODUÇÃO}

studos experimentais realizados por diferentes auto- res evidenciaram que o tratamento da dor pré-operatória, antes da incisão cirúrgica, poderia prevenir a hiperexcitabilidade dos neurônios da medula espinhal secundária à estimulação periférica, que está relacionada à hiperalgesia. Os proponentes desta técnica posteriormente, chamada analgesia preemptiva, também descobriram uma diminuição marcante na intensidade da duração da dor pós-operatória ${ }^{1,2}$. 
Os neurotransmissores excitatórios atuando através de receptores N-Metil-d aspartato (NMDA) têm sido relacionados ao desenvolvimento e à manutenção de estados patológicos de dor após lesão tecidual, principalmente por hiperalgesia e alodínia ${ }^{3}$.

Essa observação estimulou a avaliação de antagonistas dos receptores NMDA em vários modelos de dor. Na prática clínica, a cetamina representa um dos poucos antagonistas NMDA disponíveis, apresentando ainda propriedade analgésica não relacionada ao receptor NMDA, como a ativação do sistema inibitório descendente monoaminérgico, envolvido na modulação de processos nociceptivos e geralmente ativados por opióides sistêmicos ${ }^{4,5}$

Mais recentemente, foi disponibilizado comercialmente o isômero levógiro da cetamina, a S(+)cetamina, que apresenta propriedades semelhantes à forma racêmica, com menos efeitos colaterais ${ }^{6}$.

Vários estudos têm sido realizados usando anestésicos locais, opióides, antiinflamatórios e medicações como a cetamina, por diferentes vias de administração, para obtenção de efeito preemptivo, porém os resultados têm sido conflitantes 7

O objetivo deste estudo foi verificar se a injeção peridural de $\mathrm{S}(+)$ cetamina associada à bupivacaína em pacientes submetidas a histerectomia total abdominal é capaz de causar efeito analgésico preemptivo.

\section{MÉTODO}

Após a aprovação do estudo pelo Comitê de Ética Médica e assinatura do Termo de consentimento, foram estudadas 30 pacientes do sexo feminino, adultas, estado físico ASA I ou II, submetidas à histerectomia total abdominal eletiva. Todas as pacientes foram operadas no Hospital Materno Infantil da Universidade Federal do Maranhão - UFMA.

O estudo foi aleatório e encoberto, em dois grupos de igual tamanho. Os grupos (1 ou 2) foram assinalados em envelopes lacrados, preparados antes do início do estudo e abertos aproximadamente uma hora antes do ato anestésico, contendo a explicação de todo o procedimento a ser tomado pelo anestesiologista que realizaria a anestesia e que não tinha envolvimento adicional com a paciente. O pesquisador responsável não tinha conhecimento do grupo sorteado até o final do estudo.

Foram excluídas do estudo as pacientes que se recusaram a se submeter ao procedimento e as que apresentavam contra-indicações para realização de punção peridural, como infecção no local da punção, hipovolemia não corrigida, distúrbios de coagulação, anormalidades anatômicas, dificuldades técnicas e as pacientes portadoras de doenças cardíacas, coronarianas e hipertensivas.

As pacientes do Grupo 1 (grupo pré-incisional) $(n=15)$ receberam, por via peridural, $17 \mathrm{~mL}$ de bupivacaína a $0,25 \%$, sem vasoconstritor, associadas a $30 \mathrm{mg}$ de $(\mathrm{S}+)$ cetamina $(3 \mathrm{~mL})$, trinta minutos antes da incisão cirúrgica e, após trinta minutos do início da incisão, receberam $20 \mathrm{~mL}$ de solução fisiológica a $0,9 \%$.
As pacientes do Grupo 2 (grupo pós-incisional) $(n=15)$ receberam, por via peridural, $20 \mathrm{~mL}$ de solução fisiológica a $0,9 \%$, trinta minutos antes da incisão, sendo feita administração de $17 \mathrm{~mL}$ de bupivacaína a $0,25 \%$, sem vasoconstritor, associados a $30 \mathrm{mg}$ de $(\mathrm{S}+)$ cetamina $(3 \mathrm{~mL})$, trinta minutos após a incisão.

A monitorização durante a anestesia foi realizada com cardioscópio, oxímetro de pulso e monitor de pressão arterial não-invasiva. A hidratação foi feita com solução de Ringer, após punção venosa com cateter de teflon 18G. As pacientes não receberam medicação pré-anestésica.

A punção peridural foi realizada entre $L_{1}-L_{2}$, por meio da técnica da perda de resistência ao ar, seguida de injeção das soluções já descritas, passagem e fixação de cateter.

Após a injeção peridural, todas as pacientes foram submetidas à anestesia geral. A indução foi obtida com propofol $(2,5$ $\mathrm{mg} \cdot \mathrm{kg}^{-1}$ ) e o bloqueio neuromuscular com pancurônio $\left(0,1 \mathrm{mg} \cdot \mathrm{kg}^{-1}\right)$. Após a intubação orotraqueal, a anestesia foi mantida com $\mathrm{O}_{2}$ e isoflurano.

Ao término da operação, as pacientes foram observadas na sala de recuperação pós-anestésica, e posteriormente, encaminhadas para a enfermaria. Foram analisadas as necessidades de analgésicos em todos os casos nas primeiras 24 horas do pós-operatório, sendo administrada uma solução composta de $4 \mathrm{~mL}$ de bupivacaína a $0,25 \%$, sem vasoconstritor, e $1 \mathrm{~mL}$ de fentanil $(50 \mu \mathrm{g})$ pelo cateter peridural, assim que a paciente referisse dor (a partir do momento que recobrava a consciência), em intervalo mínimo de quatro horas. A equipe de enfermagem foi treinada especificamente para esse fim e todas as pacientes receberam explicações prévias de que deveriam solicitar analgésico, mesmo que a dor fosse de leve intensidade. Quando houve necessidade de complementação analgésica antes do período de 4 horas, foi administrada dipirona, na dose de $30 \mathrm{mg} \cdot \mathrm{kg}^{-1}$, por via venosa. A quantidade de analgésico necessária nas 24 horas foi registrada.As avaliações da dor foram feitas através da escala verbal e da escala numérica nos seguintes momentos:

(M0) - ao despertar; (M6) - 6 horas após o término da operação;(M12) - 12 horas após o término da operação;(M18) - 18 horas após o término da operação;(M24) - 24 horas após o término da operação.

Foram comparadas as seguintes variáveis entre os dois grupos: idade, peso, altura, índice de massa corpórea (IMC), tempo de duração das operações e intercorrências anestésico-cirúrgicas. No pós-operatório, foram comparados, entre os dois grupos, os seguintes parâmetros: avaliação de dor através de escala verbal e numérica, o tempo necessário para solicitar pela primeira vezo analgésico, o consumo total de analgésicos e a ocorrência de efeitos colaterais e possíveis complicações.Para a análise estatística dos resultados foram utilizados testes paramétricos e não paramétricos, levando-se em consideração a natureza das variáveis estudadas. Foram aplicados os seguintes testes: $t$ de Student, de Mann-Whitney, de Kolmogorov-Smirnov, do Qui-quadrado para as tabelas de contingência e Análise de Variância por Postos de Friedman.Apesar de terem sido utilizados testes 
não paramétricos, foram colocados a média e o desvio-padrão nas tabelas com o objetivo de se dar uma idéia acerca da variabilidade dos valores. Para todos os testes, fixou-se em 0,05 ou $5 \%(p \leq 0,05)$ o nível de rejeição da hipótese de nulidade.

\section{RESULTADOS}

Os dados demográficos (idade, peso, altura e índice de massa corpórea) das 30 pacientes estão expressos através da média \pm desvio padrão na tabela I. A análise estatística não demonstrou diferença significante entre os grupos 1 e 2 . O tempo total de operação variou de 50 a 115 minutos (média \pm $\mathrm{DP}: 87,66 \pm 17,40 \mathrm{~min}$ ) para o grupo pré-incisional (G1) e de 55 a 136 minutos $(76,66 \pm 25,32 \mathrm{~min})$ para o grupo pós-incisional (G2), não apresentando diferença estatisticamente significante $(p=0,0889)$.

Tabela I - Dados Demográficos das Pacientes (Média $\pm \mathrm{DP}$ )

\begin{tabular}{lcccc}
\hline & Idade (anos) & Peso $(\mathrm{kg})$ & Altura $(\mathrm{cm})$ & $\mathrm{IMC}\left(\mathrm{kg} / \mathrm{m}^{2}\right)$ \\
\hline $\mathrm{G} 1$ & $37,80 \pm 5,51$ & $54,23 \pm 8,89$ & $155,33 \pm 5,47$ & $22,39 \pm 2,71$ \\
$(\mathrm{n}=15)$ & & & & \\
$\mathrm{G} 2$ & $40,07 \pm 5,76$ & $61,87 \pm 11,31$ & $155,80 \pm 11,23$ & $25,53 \pm 4,05$ \\
$(\mathrm{n}=15)$ & & & & \\
$\mathrm{p}$ & $0,2055 \mathrm{~ns}$ & $0,0493 \mathrm{~ns}^{1}$ & $0,8860 \mathrm{~ns}^{2}$ & $0,0102 \mathrm{~ns}^{3}$
\end{tabular}

G1: grupo pré-incisional; G2: grupo pós-incisional; p: significância estatística, $\leq$ 0,05; IMC: índice de massa corpórea; ns: não significativo teste de Mann-Whitney; ns1: não significativo - teste $t$ Student (corrigido pela Análise de Co-Variância); $\mathrm{ns}^{2}$ : não significativo - teste $t$ Student ; $\mathrm{ns}^{3}$ : não significativo - teste de Mann-Whitney (corrigido pela Análise de Co-Variância)

O tempo necessário para solicitar pela primeira vez a complementação analgésica variou de 0 a 185 minutos (mediana: $38,33 \mathrm{~min}$ ) para o grupo 1 , e de 0 a 270 minutos (mediana: $58,33 \mathrm{~min}$ ) para o grupo 2 , não apresentando diferença significante $(p=0,3712)$.

Em relação ao número de bolus de solução analgésica peridural (bupivacaína a 0,25\% - $4 \mathrm{~mL}+$ fentanil $50 \mu \mathrm{g}-1 \mathrm{~mL}$ ) e ao número de complementações com dipirona (30 mg) por via venosa solicitadas por paciente, não houve diferença significante entre os dois grupos, de acordo com os dados apresentados na tabela II.
Tabela II - Analgesia Pós-Operatória: Número de Bolus de Solução Analgésica Peridural e Número de Complementação com Dipirona Venosa Solicitados por Paciente (Média $\pm \mathrm{DP}$ )

\begin{tabular}{lcc}
\hline & $\begin{array}{c}\text { Número de Bolus de } \\
\text { Solução Analgésica } \\
\text { Peridural }\end{array}$ & $\begin{array}{c}\text { Número de } \\
\text { Complementações com } \\
\text { Dipirona Venosa }\end{array}$ \\
\hline $\begin{array}{l}\mathrm{G} 1 \\
(\mathrm{n}=15)\end{array}$ & $4,46 \pm 1,05$ & $1,53 \pm 091$ \\
$\mathrm{G} 2$ & $\mathrm{n}=15)$ & $1,33 \pm 0,81$ \\
$\mathrm{p}$ & $4,40 \pm 1,05$ & $0,5435 \mathrm{~ns}$ \\
\hline
\end{tabular}

G1: grupo pré-incisional; G2: grupo pós-incisional; p: significância estatística, $p \leq 0,05$; ns: não significativo - Teste de Mann-Whitney; valores expressos em Média \pm DP

Também não houve diferença no número total de bolus de solução analgésica peridural e no número total de complementações com dipirona $(30 \mathrm{mg}$ ) por via venosa, acumulados em relação aos seguintes intervalos: M0 - M6, M6 - M12, M12 M18 e M18-M24 horas, conforme demonstrado na tabela III. Quando foram comparados os escores referentes à intensidade da dor pela escala numérica e pela escala verbal entre os dois grupos, não foi observada diferença estatisticamente significantes nos valores médios da intensidade da dor, conforme mostra a figura 1 e a tabela IV, respectivamente.

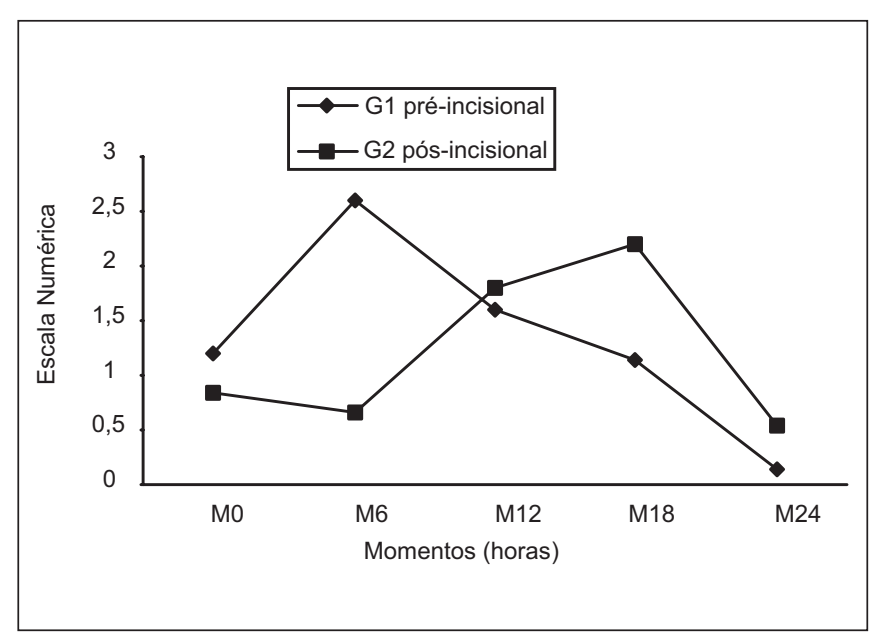

Figura 1 - Valores Médios da Escala Numérica em Relação aos Momentos, Grupos 1 e 2

Tabela III - Analgesia Pós-Operatória: Número Total de Bolus de Solução Analgésica Peridural e o Número Total de Complementações com Dipirona Acumulados em Relação aos Intervalos de Momento

\begin{tabular}{|c|c|c|c|c|c|c|c|c|}
\hline & \multicolumn{4}{|c|}{ Número Total de Bolus } & \multicolumn{4}{|c|}{ Número Total de Complementações com Dipirona } \\
\hline & $\mathrm{M} 0-\mathrm{M} 6$ & M6 -M12 & M12-M18 & M18-M24 & $\mathrm{M} 0-\mathrm{M} 6$ & M6 -M12 & M12-M18 & M18-M24 \\
\hline $\begin{array}{l}\text { G1 } \\
(n=15)\end{array}$ & 20 & 34 & 51 & 67 & 11 & 14 & 19 & 23 \\
\hline $\begin{array}{l}G 2 \\
(n=15)\end{array}$ & 22 & 33 & 49 & 66 & 9 & 14 & 18 & 20 \\
\hline p & \multicolumn{4}{|c|}{ ns } & \multicolumn{4}{|c|}{ ns } \\
\hline
\end{tabular}

G1: grupo pré-incisional; G2: grupo pós-incisional; $p$ : significância estatística, $p \leq 0,05$; ns: não significativo - Teste de Kolmogorov-Smirrnov 
Tabela IV - Valores Mensurados pela Escala Verbal nos Dois Grupos em Cada Momento

\begin{tabular}{|c|c|c|c|c|c|}
\hline \multirow[t]{2}{*}{ Momentos } & \multirow[t]{2}{*}{ Intensidade } & \multicolumn{2}{|c|}{ Grupo 1} & \multicolumn{2}{|c|}{ Grupo 2} \\
\hline & & $\mathrm{n}=15$ & $\%$ & $n=15$ & $\%$ \\
\hline M0 & Ausente & 12 & 80 & 12 & 80 \\
\hline \multirow[t]{3}{*}{$p=0,7212$} & Leve & 1 & 6,7 & 2 & 13,3 \\
\hline & Moderado & 1 & 6,7 & 0 & 0 \\
\hline & Intenso & 1 & 6,7 & 1 & 6,7 \\
\hline M6 & Ausente & 10 & 66,7 & 14 & 93,3 \\
\hline \multirow[t]{3}{*}{$p=0,1599$} & Leve & 0 & 0 & 0 & 0 \\
\hline & Moderado & 2 & 13,3 & 0 & 0 \\
\hline & Intenso & 3 & 20 & 1 & 6,7 \\
\hline M12 & Ausente & 11 & 73,3 & 10 & 66,7 \\
\hline \multirow[t]{3}{*}{$p=0,3843$} & Leve & 0 & 0 & 2 & 13,3 \\
\hline & Moderado & 3 & 20 & 3 & 20 \\
\hline & Intenso & 1 & 6,7 & 0 & 0 \\
\hline M18 & Ausente & 12 & 80 & 9 & 60 \\
\hline \multirow[t]{3}{*}{$p=0,2514$} & Leve & 1 & 6,7 & 1 & 6,7 \\
\hline & Moderado & 1 & 6,7 & 5 & 33,3 \\
\hline & Intenso & 1 & 6,7 & 0 & 0 \\
\hline M24 & Ausente & 14 & 93,3 & 13 & 86,7 \\
\hline \multirow[t]{3}{*}{$p=0.5954$} & Leve & 1 & 6,7 & 1 & 6,7 \\
\hline & Moderado & 0 & 0 & 1 & 6,7 \\
\hline & Intenso & 0 & 0 & 0 & 0 \\
\hline
\end{tabular}

G1: grupo pré-incisional; G2: grupo pós-incisional; teste do Qui-quadrado p s 0,05; M0 ,M6, M12, M18, M24: G1 = G2

Em relação às intercorrências, foram observados: hipotensão arterial (uma paciente em cada grupo), hipertensão arterial (G1 = uma, G2 = três pacientes), sangramento (uma paciente no G2), disritmia (G1 = uma paciente, G2 = duas pacientes) e vômitos (três pacientes em cada grupo). Não foi observado nenhum caso de alteração do nível de consciência.

\section{DISCUSSÃo}

Vários estudos foram realizados sobre analgesia preemptiva, mas o método de muitos deles não segue o modelo de uma intervenção que, feita antes do início do estímulo doloroso, tenha efeito significativamente maior, menor ou igual quando comparada com a mesma intervenção feita após o início da dor, pela mesma via e com a mesma dose.

Este estudo foi realizado de acordo com uma proposta analgésica em que fármacos foram administrados antes e depois do início do estímulo doloroso, na mesma dose e pela mesma via, conforme descrito por Mcquay e considerado por vários autores como modelo de analgesia preemptiva ${ }^{8,9}$.

Foram avaliados dois grupos de pacientes do sexo feminino, submetidos à histerectomia total abdominal, operadas pela mesma equipe cirúrgica, sob a mesma técnica anestésica, não havendo diferença estatisticamente significativa para idade, peso, altura e IMC. A duração total da operação também foi semelhante para os dois grupos.

A histerectomia total abdominal foi escolhida por ser uma operação de médio porte que causa estímulos nociceptivos intensos e por estar comumente associada a dor de forte intensidade no período pós-operatório.

Na indução e manutenção da anestesia foram utilizados, respectivamente, propofol e isoflurano que são fármacos que não estão relacionados a efeito preemptivo, segundo vários autores $^{10-12}$.

Devido à possibilidade de prevenção ou atenuação de sensibilização central com uso de opióides, evitou-se o emprego de tais fármacos neste estudo, que poderiam levar a uma interpretação duvidosa dos resultados ${ }^{13-16}$.

Uma dose sub-anestésica de cetamina definida como uma administração em bolus menor que $1 \mathrm{mg} \cdot \mathrm{kg}^{-1}$, pelas vias venosa ou peridural, foi relacionada a efeitos analgésicos em contraste com uma dose maior que possui sintomas psicomiméticos e anestesia dissociativa ${ }^{17}$. Os autores concluíram que doses sub-anestésicas de cetamina em associação com anestésicos locais ou opióides melhoram significativamente os escores de dor, reduzindo a necessidade de outras medicações e a área de hiperalgesia ao redor da ferida operatória. A cetamina pode agir em vários sistemas receptores, como os opióides $(\mu, \delta, \kappa)$ e os colinérgicos (muscarínicos e nicotínico), envolvendo o sistema monoaminérgico e exibindo também efeitos anestésicos locais, pelo bloqueio dos canais de sódio ${ }^{18-20}$. Todas essas interações provavelmente podem mediar efeitos analgésicos não especificamente relacionados com o sistema NMDA e contribuir no alívio da dor pós-operatória, após a administração peridural. A cetamina possui um efeito anti-hiperalgesia. Em estudo experimental, 
Kawamata e col. ${ }^{21}$ esclareceram parte dos efeitos analgésicos da cetamina. Os autores mediram os efeitos da cetamina pelas vias intraperitoneal e subaracnóidea em ratos com e sem inflamação. O envolvimento do sistema monoaminérgico foi avaliado através da administração de antagonistas específicos e técnicas de microdiálise de líquor. $\mathrm{Na}$ ausência de reação inflamatória, a cetamina produziu efeito antinociceptivo através da ativação do sistema inibitório monoaminérgico descendente. Este efeito ocorreu somente após a administração sistêmica. Entretanto, a absorção de cetamina do espaço peridural para a circulação sistêmica é rápida, não sendo possível descartar que, por esta via, não haja um efeito supra-espinhal. Nos ratos que apresentaram inflamação periférica, os efeitos antinociceptivos da cetamina são claramente independentes do sistema monoaminérgico e são devidos a um efeito anti-NMDA. Nesta situação a antinocicepção ocorre independente da via de administração ${ }^{21}$.

A cetamina racêmica não é recomendada para uso peridural e subaracnóideo, por causa do potencial neurotóxico do conservante que é associado à sua preparação comercial ${ }^{22}$.

A S(+)cetamina deste estudo foi produzida em uma solução sem conservante e tem se mostrado superior à mistura racêmica sob vários aspectos. Apresenta afinidade quatro vezes maior pelo receptor NMDA em relação à forma $R(-)$ e potência analgésica maior que a forma racêmica ${ }^{23,24}$. Também têm sido descritos menores percentuais de efeitos colaterais, como agitação e reações psicóticas ${ }^{6}$.

Alguns estudos foram publicados com o uso peridural de $S(+)$ cetamina e recentemente foi descrita sua administração subaracnóidea em infusão contínua por 24 dias em paciente com dor crônica, sem lesão neurológica ${ }^{25-27}$.

No presente estudo foi utilizada a dose de $30 \mathrm{mg}$ por via peridural, considerada sub-anestésica, mas os resultados indicam que a cetamina por esta via não foi capaz de proporcionar analgesia preemtiva, pois os escores de dor na escala numérica e verbal, o tempo para solicitação de analgésico pela primeira vez e o consumo de analgésico pós-operatório nos grupos pré e pós-incisionais não foram significativamente diferentes.

Por que a cetamina peridural não foi eficaz nesse estudo? Várias explicações podem ser propostas. Primeiro, no segmento espinhal, os receptores NMDA têm um importante papel no processamento da percepção da dor. Particularmente esses receptores prolongam e amplificam a resposta nociceptiva. Todos os pacientes receberam bloqueio peridural com anestésico local antes ou durante o ato cirúrgico, o que pode ter interferido na ação da cetamina peridural nos receptores NMDA. A cetamina induz um bloqueio não competitivo do receptor NMDA, que faz com que o canal do receptor tenha que estar em estado aberto antes que o fármaco se ligue a ele. Um bloqueio peridural que produz inibição pré-sináptica dos receptores nociceptivos aferentes poderia ter evitado que o receptor NMDA ficasse no estado aberto ${ }^{3}$.

Segundo, como sugerido por alguns autores, o papel dos antagonistas NMDAna dor secundária à incisão cirúrgica poderia ser menos importante que em outros modelos experimen- tais de dor. Os autores investigaram os efeitos dos antagonistas dos receptores NMDA na hiperalgesia mecânica em um modelo de dor pós-operatória e falharam em demonstrar um efeito espinhal significativo ${ }^{28}$.

Terceiro, existe um local mais central para a ação anti-hiperalgésica da cetamina, que é facilmente alcançado após a administração sistêmica. Também a cirurgia intraperitoneal ativa estímulos aferentes nociceptivos (ramos sensitivos do nervo vago) e, finalmente, a cetamina interfere diretamente com a reação inflamatória. Autores demonstraram in vitro um potente efeito inibidor da cetamina em várias citocinas pró-inflamatórias ${ }^{29}$. Nessas duas últimas situações cetamina sistêmica é provavelmente mais eficiente que a cetamina peridural.

Em relação ao uso da cetamina no espaço peridural, para determinar efeito preemptivo os dados são limitados. Schmid e col. ${ }^{17}$ evidenciaram um efeito promissor da cetamina por várias vias, inclusive a peridural. Choe e col. ${ }^{30}$ associando cetamina e morfina peridural, em pacientes submetidos à gastrectomia e Wong e col. ${ }^{31}$, em pacientes que realizaram prótese de joelho, obtiveram efeito preemptivo.

Revisão sistemática de oitenta estudos sobre analgesia preemptiva foi feita por Moiniche, Kehlet, Dahl ${ }^{32}$, incluindo AINH, opióides por via venosa, antagonista dos receptores NMDA por via parenteral (venosa e muscular), analgesia peridural (doses simples ou infusão contínua), analgesia caudal e anestesia local. Os estudos usando cetamina venosa foram uniformemente negativos. Apenas dois estudos utilizando dextrometorfano, outro fármaco com propriedades antagonistas NMDA, foram efetivos em obter efeitos preemptivos.

Entretanto, vale ressaltar que foram apenas seis estudos com cetamina e parece ser mais apropriado dizer que os resultados são ainda inconclusivos.

Em relação à cetamina peridural isolada ou em associação, apenas dois estudos foram incluídos, não sendo possível chegar-se a uma definição de que, por esta via e com este fármaco, não seja evidenciado efeito preemptivo.

O tratamento da dor com peridural contínua para determinar efeito preemptivo estendendo-se até o período pós-operatório também foi levantado. Teoricamente este tratamento pode melhorar a capacidade de reduzir os impulsos nociceptivos e a neuroplasticidade central, estes sendo causados não apenas pela incisão e procedimento cirúrgico, como também pela inflamação pós-cirúrgica. Entretanto os resultados obtidos demonstraram que o efeito preemptivo foi sistematicamente negativo, valendo a ressalva de que foram apenas oito estudos. Em pequeno número de casos foi observada melhora na intensidade da dor. Uma explicação para os achados negativos da utilização da peridural contínua pode ser que, apesar do tratamento continuado, a técnica tenha sido insuficiente para prevenir o desencadeamento e manutenção da lesão induzida pela sensibilização central. É inteiramente aceito que a analgesia preemptiva possa reduzir o risco de desenvolver a dor pós-operatória crônica. $\mathrm{Na}$ tentativa de comparar os efeitos de tratamentos idênticos pré e pós- incisional em períodos mais longos de dor, alguns au- 
tores observaram que a percentagem de pacientes com dor seis meses depois da cirurgia foi significativamente reduzida ${ }^{32}$. Contudo, é óbvio que são necessários mais dados e novas tentativas de tratamento preemptivo comparando pacientes que não receberam nenhum tratamento em cirurgias como prostatectomias, toracotomias, ou amputações.

Neste estudo foi utilizado um volume de $17 \mathrm{~mL}$ de bupivacaína a $0,25 \%$ associados a $3 \mathrm{~mL}(30 \mathrm{mg})$ de $\mathrm{S}(+)$ cetamina. Trata-se de volume de solução analgésica utilizada com freqüência por via peridural associada à anestesia geral, que promove estabilidade anestésica sem causar alterações cardiovasculares. Entretanto, não foi avaliado o dermátomo atingido pela injeção peridural e talvez esse volume $(20 \mathrm{~mL})$ não tenha sido suficiente para bloquear completamente os estímulos aferentes nociceptivos oriundos da intervenção cirúrgica. É importante lembrar que a analgesia somática adequada não traduz necessariamente analgesia visceral. Para a analgesia pós-operatória foram administrados $5 \mathrm{~mL}$ de bupivacaína a $0,25 \%$ associados com $50 \mu \mathrm{g}$ de fentanil em bolus, a partir do momento da referência da dor até 24 horas do pós-operatório. O uso em bolus foi feito sob demanda, com intervalo mínimo de 4 horas, mas com injeção feita imediatamente após a solicitação, tentando evitar-se ao máximo quaisquer retardos na administração da solução analgésica e aumento na intensidade da dor. A quantidade de analgésicos complementares usadas foi uma maneira de avaliar a analgesia preemptiva. Não foi utilizada bomba de infusão do tipo analgesia controlada pelo paciente (ACP), devido aos custos impostos e por não ser uma técnica de rotina aplicada no hospital onde foi realizado o estudo.

Foi feita complementação analgésica com dipirona 30 $\mathrm{mg} \cdot \mathrm{kg}^{-1}$ nos pacientes que solicitaram analgésicos antes de um intervalo de 4 horas. A dipirona tem excelente ação analgésica e não está associada a risco de sangramento no pós-operatório, pois não causa alteração significativa no tempo de tromboplastina nem na agregação plaquetária e ainda tem a vantagem de ter ação espasmolítica. No pós-operatório reduz o consumo de morfina, promovendo a analgesia adequada. Adicionalmente, há evidências de que a dipirona está implicada na inibição da sensibilização central e periférica ${ }^{33-36}$.

Vale a pena ressaltar que os escores de dor em todos os tempos foram baixos, propiciando analgesia de boa qualidade com o uso da técnica proposta.

As intercorrências observadas com o emprego da conduta analgésica estudada, bem como da técnica de anestesia foram leves, não causando a exclusão das pacientes do estudo.

Não foram encontrados estudos em analgesia preemptiva na literatura que usassem $\mathrm{S}(+)$ cetamina via peridural comparando o uso pré e pós-incisional e em associação com bupivacaína. Este estudo investiga pela primeira vez a analgesia obtida com a associação peridural de $\mathrm{S}(+)$ cetamina e bupivacaína no modelo adotado.

Recentemente, Kissin ${ }^{37}$ relatou que uma abordagem analgésica preemptiva ideal deve evitar o estabelecimento da sensibilização central pela incisão cirúrgica e pelo processo inflamatório no período pós-operatório inicial. Talvez a plasticidade neuronal induzida nesse período seja a principal razão pela qual inúmeros estudos comparativos pré e pós-incisionais tenham falhado em avaliar o valor da analgesia preemptiva. Este modelo é bom o suficiente para assegurar a ocorrência ou não do efeito, mas não para mensurar o seu valor clínico. No grupo pré-incisional podem ser gerados impulsos capazes de iniciar hipersensibilização durante o período pós-cirúrgico e daí haveria uma redução da diferença entre os grupos. Ainda, o intervalo de tempo da administração dos analgésicos entre os grupos resulta em maiores concentrações plasmáticas desses medicamentos no grupo pós-incisional, o que pode causar efeitos preventivos da hipersensibilização mais pronunciados neste grupo e, conseqüentemente, reduzir a diferença entre os resultados. Um aspecto importante que também deve ser revisto nos estudos de analgesia preemptiva é a ausência de verificação da efetividade do efeito farmacológico do tratamento, através da mensuração indireta do grau da resposta inicial nociceptiva entre os grupos preemptivo e controle, medido por exemplo, pela dosagem plasmática de cortisol, de $\beta$-endorfinas, interleucinas, etc. Neste campo, pode ser citado um estudo realizado em pacientes submetidas a histerectomia abdominal com bupivacaína e fentanil peridural, que revelou que o uso de solução analgésica, previamente à incisão cirúrgica, não modificou significativamente o aumento dos níveis séricos de interleucina-6, importante mediador da fase aguda da reação inflamatória e responsável por várias das alterações endócrinas e metabólicas observadas na resposta ao trauma cirúrgico ${ }^{38}$.

Concluindo, apesar do uso de uma técnica combinada (bupivacaína + S(+)cetamina peridural) neste estudo apresentar um resultado negativo, fica a certeza de que mais pesquisas devem ser conduzidas e novas associações devem ser testadas, inclusive utilizando outros antagonistas de receptores NMDA, em um modelo de estudo mais prolongado, desde antes da incisão cirúrgica até o período pós-operatório imediato, na tentativa de obter atenuação das respostas que prolongam e acentuam a dor, com cobertura analgésica mais ampla durante todo esse período.

\section{Preemptive Analgesia with Epidural Bupivacaine and $S(+) K e t a m i n e$ in Abdominal Hysterectomy}

Ferdinand Edson de Castro, M.D.; João Batista Santos Garcia, TSA, M.D.

\section{INTRODUCTION}

Some experimental dates by different authors have shown that preoperative pain treatment before surgical incision could prevent spinal cord neuronal hyperexcitability second- 
ary to peripheral stimulation, which is related to hyperalgesia. Proponents of this technique, later called preemptive analgesia, have also found a marked decrease in postoperative pain length ${ }^{1,2}$.

Excitatory neurotransmitters, acting through N-Methyl-D aspartate (NMDA), have been related to the development and maintenance of pathological pain states after tissue injury, especially hyperalgesia and allodynia ${ }^{3}$.

This observation has encouraged the evaluation of NMDAreceptor antagonists in different pain models. Ketamine is one of the few NMDA antagonists available for clinical practice. In addition, it has analgesic properties unrelated to NMDA receptors, such as activation of descending monoaminergic inhibitory system involved in the modulation of nociceptive processes which are in general activated by systemic opioids ${ }^{4,5}$. Several studies have been performed with local anesthetics, opioids, anti-inflammatory drugs and medications such as ketamine by different administration routes to obtain preemptive effects, however with conflicting results ${ }^{7}$.

This study aimed at checking whether epidural injection of $\mathrm{S}(+)$ ketamine plus bupivacaine in patients submitted to total abdominal hysterectomy would have preemptive analgesic effect.

\section{METHODS}

After the Medical Ethics Committee approval and their written consent, participated in this study 30 female adult patients, physical status ASA I or II, submitted to elective total abdominal hysterectomy. All patients were operated in the Hospital Materno Infantil, Universidade Federal do Maranhão (UFMA).

This was a randomized, double-blind study with two equal groups. Groups (1 or 2) were assigned to sealed envelopes prepared before beginning of study and opened approximately one hour before anesthesia, after explaining the whole procedure to be performed by the anesthesiologist who had no additional involvement with the patient. The researcher in charge was blind to the drawn group until study completion.

Exclusion criteria were counterindication for epidural puncture, such as infection at puncture site, uncorrected hypovolemia, patient's refusal, coagulation disorders, anatomic abnormalities, technical difficulties and patients with cardiac, coronary or hypertensive diseases.

Group I patients (pre-incision group) $(n=15)$ received epidural $17 \mathrm{~mL}$ of $0.25 \%$ bupivacaine plus $30 \mathrm{mg}$ $\mathrm{S}(+)$ ketamine $(3 \mathrm{~mL}) 30$ minutes before surgical incision, and $20 \mathrm{~mL}$ saline 30 minutes after incision.

Group 2 patients (post-incision group) $(n=15)$ received epidural $20 \mathrm{~mL}$ saline 30 minutes after incision, and $17 \mathrm{~mL}$ of $0.25 \%$ bupivacaine plus $30 \mathrm{mg} \mathrm{S}(+)$ ketamine $(3 \mathrm{~mL}) 30 \mathrm{~min}-$ utes after incision.

Monitoring during anesthesia consisted of cardioscope, pulse oximetry and noninvasive blood pressure. Hydration was achieved with lactated Ringer's after venous puncture with Teflon $18 \mathrm{G}$ catheter. Patients were not premedicated.
Epidural puncture was performed at $L_{1}-L_{2}$ interspace through loss of resistance to air technique followed by above-described solutions injection, catheter insertion and fixation. All patients were submitted to general anesthesia after epidural injection. Anesthesia was induced with propofol (2.5 $\left.\mathrm{mg} . \mathrm{kg}^{-1}\right)$ and neuromuscular block with pancuronium (0.1 $\left.\mathrm{mg} \cdot \mathrm{kg}^{-1}\right)$. After tracheal intubation anesthesia was maintained with $\mathrm{O}_{2}$ and isoflurane.

At the end of surgery, patients were observed in the PACU and then referred to the ward. Need for analgesics in all cases during the first 24 hours was evaluated, being administered 4 $\mathrm{mL}$ of $0.25 \%$ bupivacaine and $1 \mathrm{~mL}$ fentanyl $(50 \mu \mathrm{g})$ via epidural catheter as soon as patient would refer pain (as from emergence), in minimum intervals of 4 hours. Nursing team was specifically trained for this purpose and all patients were previously informed that they should ask for analgesics even for mild pain. Intravenous $30 \mathrm{mg} \cdot \mathrm{kg}^{-1}$ dipyrone was administered when there was need for analgesic supplementation before 4 hours. Total analgesic consumption in 24 hours has been recorded.Pain was evaluated by verbal scale and numeric scale in the following moments:

(M0) - at emergence; (M6) - 6 hours after the end of surgery; (M12) - 12 hours after the end of surgery; (M18) - 18 hours after the end of surgery; (M24) - 24 hours after the end of surgery.

The following variables were compared between groups: age, weight, height, body mass index (BMI), surgery length and intercurrences. The following parameters were evaluated after surgery: pain evaluation through verbal and numeric scale, time elapsed for first analgesic request, total analgesic consumption, side effects and complications. Parametric and non-parametric tests were used for statistical analysis of results, taking into consideration the nature of studied variables. The following tests were applied: Student's $t$, Mann-Whitney, Kolmogorov-Smirnov, chi-square for contingency tables and Friedman's Analysis of Variance by Posts. Notwithstanding the application of non-parametric tests, tables show mean and standard deviation to give an Idea of values variability. Null hypothesis rejection level was set to 0.05 or $5 \%(p \leq 0.05)$ for all tests.

\section{RESULTS}

Demographics data (age, weight, height and body mass index) are shown in table I and are expressed in mean \pm standard deviation. Statistical analysis has not shown significant differences between groups. Total surgery length has varied from 50 to 115 minutes (mean \pm SD: $87.66 \pm 17.40 \mathrm{~min}$ ) for the pre-incision group (G1) and from 55 to 136 minutes (76.66 \pm $25,32 \mathrm{~min}$ ) for the post-incision group (G2) without statistically significant difference $(p=0.0889)$.

Time for first analgesic supplementation has varied from 0 to 185 minutes (median: $38.33 \mathrm{~min}$ ) for group 1 and from 0 to 270 minutes (median: $58.33 \mathrm{~min}$ ) for group 2, without significant difference $(p=0.3712)$. 
Table I - Demographics Data (Mean \pm SD)

\begin{tabular}{lcccc}
\hline & Age (years) & Weight $(\mathrm{kg})$ & Height $(\mathrm{cm})$ & $\mathrm{BMl}\left(\mathrm{kg} / \mathrm{m}^{2}\right)$ \\
\hline $\mathrm{G} 1$ & $37.80 \pm 5.51$ & $54.23 \pm 8.89$ & $155.33 \pm 5.47$ & $22.39 \pm 2.71$ \\
$(\mathrm{n}=15)$ & & & & \\
$\mathrm{G} 2$ & $40.07 \pm 5,76$ & $61.87 \pm 11.31$ & $155.80 \pm 11.23$ & $25.53 \pm 4.05$ \\
$(\mathrm{n}=15)$ & & & & \\
$\mathrm{p}$ & $0.2055 \mathrm{~ns}$ & $0.0493 \mathrm{~ns}^{1}$ & $0.8860 \mathrm{~ns}^{2}$ & $0.0102 \mathrm{~ns}^{3}$ \\
\hline
\end{tabular}

G1: pre-incision group; G2: post-incision group; $P$ : statistical significance, $\leq$ 0.05; BMI: body mass index; ns: non significant - Mann-Whitney test; ns : non significant - Student's $t$ test (corrected by Analysis of Co-Variance); $\mathrm{ns}^{2}$. non significant - Student's $t$ test ns $^{3}$ : non significant - Mann-Whitney test (corrected by Analysis of Co-variance)

There have been no significant differences between groups in the number of epidural analgesic solution bolus $(0.25 \%$ bupivacaine $-4 \mathrm{~mL}+$ fentanyl $50 \mu \mathrm{g}-1 \mathrm{~mL}$ ) and the number of supplementations with intravenous dipyrone $(30 \mathrm{mg})$ requested by each patient (Table II).

Table II - Postoperative Analgesia: Number of Epidural Analgesic Solution Bolus and Number of Supplementations with Intravenous Dipyrone Requested by $\mathrm{Pa}$ tients (Mean \pm SD)

\begin{tabular}{lcc}
\hline & $\begin{array}{c}\text { Number of Epidural } \\
\text { Analgesic Solution Bolus }\end{array}$ & $\begin{array}{c}\text { Number of Supplementations } \\
\text { with Intravenous Dipyrone }\end{array}$ \\
\hline $\mathrm{G} 1$ & $4.46 \pm 1.05$ & $1.53 \pm 0.91$ \\
$(\mathrm{n}=15)$ & $4.40 \pm 1.05$ & $1.33 \pm 0.81$ \\
$\mathrm{G} 2$ & & \\
$\mathrm{n}=15)$ & $0.9166 \mathrm{~ns}$ & $0.5435 \mathrm{~ns}$ \\
$\mathrm{p}$ & & \\
\hline
\end{tabular}

G1: pre-incision group; G2: post-incision group; $P$ : statistical significance, $p$ $\leq 0.05$; ns: non significant - Mann-Whitney test

There have also been no differences in total number of epidural analgesic solution bolus and total number of supplementations with intravenous dipyrone $(30 \mathrm{mg})$ accumulated in the following moments: M0 - M6, M6 - M12, M12 M18 e M18 - M24 hours, as shown in table III.

When numeric and verbal pain scores were compared between groups, there has been no statistically significant difference in mean pain intensity, as shown in figure 1 and table IV, respectively.

The following intercurrences were observed: hypotension (one patient from each group), hypertension ( $\mathrm{G} 1=1, \mathrm{G} 2=3$ patients), bleeding (G2 = 1 patient), arrhythmia (G1 = 1 pa-

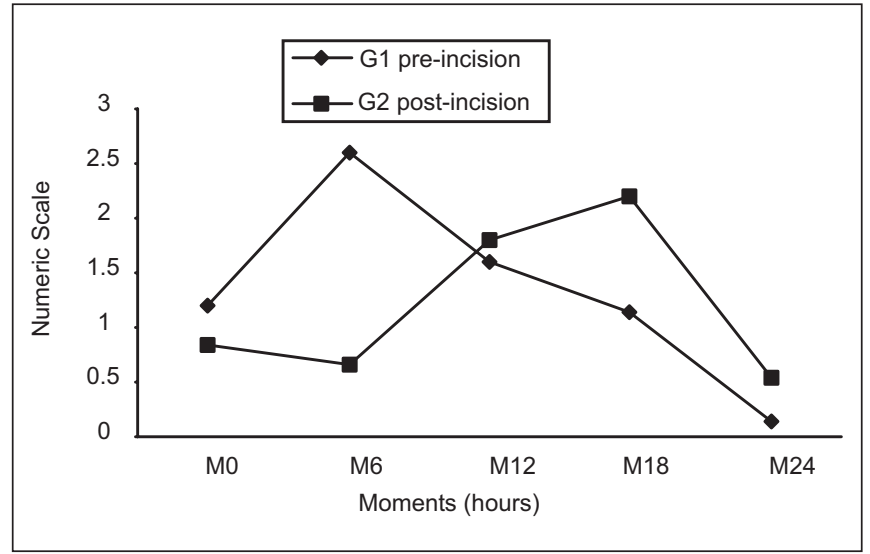

Figure 1 - Numeric Scale Mean Values in Different Moments, Groups 1 and 2

tient, G2 = 2 patients) and vomiting ( 3 patients from each group). There has been no consciousness level change.

\section{DISCUSSION}

Several studies on preemptive analgesia have been conducted, but many do not follow the model in which the intervention before painful stimulation has significantly higher, lower or equal effect as compared to the same intervention after pain stimulation by the same route and with the same dose.

Our study was performed according to an analgesic proposal in which drugs were administered before and after painful stimulation in the same dose and by the same route, as described by Mcquay, and considered the preemptive analgesia model by several authors ${ }^{8,9}$.

Participated in this study two groups of female patients submitted to total abdominal hysterectomy, operated by the same surgical team under the same anesthetic technique, without statistically significant differences in age, weight, height and BMI. Total surgery length was also similar for both groups.

Total abdominal hysterectomy was chosen for being a medium-size procedure promoting intense nociceptive stimulations, and for being commonly associated to severe postoperative pain.

Table III - Postoperative Analgesia: Total number of Epidural Analgesic Solution Bolus and Total Number of Dipyrone Supplementations Accumulated during Moment Intervals

\begin{tabular}{|c|c|c|c|c|c|c|c|c|}
\hline & \multicolumn{4}{|c|}{ Total Number of Bolus } & \multicolumn{4}{|c|}{ Total Number of Supplementations with Dipyrone } \\
\hline & M0 -M6 & M6 -M12 & M12-M18 & M18-M24 & M0 -M6 & M6 -M12 & M12-M18 & M18-M24 \\
\hline $\begin{array}{l}\mathrm{G} 1 \\
(n=15)\end{array}$ & 20 & 34 & 51 & 67 & 11 & 14 & 19 & 23 \\
\hline $\begin{array}{l}\mathrm{G} 2 \\
(\mathrm{n}=15)\end{array}$ & 22 & 33 & 49 & 66 & 9 & 14 & 18 & 20 \\
\hline $\mathrm{p}$ & \multicolumn{4}{|c|}{ ns } & \multicolumn{4}{|c|}{ ns } \\
\hline
\end{tabular}

G1: pre-incision group; G2: post-incision group; $P$ : st atistical significance, $p \leq 0.05$; ns: non significant - Kolmogorov-Smirrnov test 
Table IV - Verbal Scale Values Measured for both Groups in Each Moment

\begin{tabular}{|c|c|c|c|c|c|}
\hline \multirow[t]{2}{*}{ Moments } & \multirow[t]{2}{*}{ Intensity } & \multicolumn{2}{|c|}{ Group 1} & \multicolumn{2}{|c|}{ Group 2} \\
\hline & & $\mathrm{n}=15$ & $\%$ & $\mathrm{n}=15$ & $\%$ \\
\hline MO & Absent & 12 & 80 & 12 & 80 \\
\hline \multirow[t]{3}{*}{$p=0.7212$} & Mild & 1 & 6.7 & 2 & 13.3 \\
\hline & Moderate & 1 & 6.7 & 0 & 0 \\
\hline & Severe & 1 & 6.7 & 1 & 6.7 \\
\hline M6 & Absent & 10 & 66.7 & 14 & 93.3 \\
\hline \multirow[t]{3}{*}{$p=0.1599$} & Mild & 0 & 0 & 0 & 0 \\
\hline & Moderate & 2 & 13.3 & 0 & 0 \\
\hline & Severe & 3 & 20 & 1 & 6.7 \\
\hline M12 & Absent & 11 & 73.3 & 10 & 66.7 \\
\hline \multirow[t]{3}{*}{$p=0.3843$} & Mild & 0 & 0 & 2 & 13.3 \\
\hline & Moderate & 3 & 20 & 3 & 20 \\
\hline & Severe & 1 & 6.7 & 0 & 0 \\
\hline M18 & Absent & 12 & 80 & 9 & 60 \\
\hline \multirow[t]{3}{*}{$p=0.2514$} & Mild & 1 & 6.7 & 1 & 6.7 \\
\hline & Moderate & 1 & 6.7 & 5 & 33.3 \\
\hline & Severe & 1 & 6.7 & 0 & 0 \\
\hline M24 & Absent & 14 & 93.3 & 13 & 86.7 \\
\hline \multirow[t]{3}{*}{$p=0.5954$} & Mild & 1 & 6.7 & 1 & 6.7 \\
\hline & Moderate & 0 & 0 & 1 & 6.7 \\
\hline & Severe & 0 & 0 & 0 & 0 \\
\hline
\end{tabular}

G1: pre-incision group; G2: post-incision group; Chi-square test $p \leq 0.05$; M0, M6, M12, M18, M24: G1 = G2

Propofol and isoflurane, which are unrelated to preemptive effects according to several authors, were used for anesthetic induction and maintenance, respectively ${ }^{10-12}$.

Opioids were avoided in this study because they could have prevented or attenuated central sensitization, thus leading to questionable results ${ }^{13-16}$.

A sub-anesthetic ketamine dose, defined as intravenous or epidural bolus below $1 \mathrm{mg} \cdot \mathrm{kg}^{-1}$, was related to analgesic effects, as compared to a higher dose with psychomimetic symptoms and dissociated analgesia ${ }^{17}$. Authors have concluded that sub-anesthetic ketamine doses associated to local anesthetics or opioids significantly improve pain scores decreasing the need for other drugs and the hyperalgesic area around the surgical wound.

Ketamine may act on several receptor systems, including opioids $(\mu, \delta, \kappa)$ and cholinergics (muscarinic and nicotinic), involving the monoaminergic system and also exhibiting local anesthetic effects by blocking sodium channels ${ }^{18-20}$. All those interactions are likely to mediate analgesic effects not specifically related to NMDA system, and may contribute to postoperative pain relief after epidural administration. Ketamine has anti-hyperalgesic effect.

Kawamata et al. ${ }^{21}$ have explained in an experimental study part of ketamine analgesic effects. Authors have measured intraperitoneal and subarachnoid ketamine effects in rats with and without inflammation. The involvement of the monoaminergic system was evaluated through the administration of specific antagonists and CSF microdialysis techniques. In the absence of inflammatory reaction, ketamine has promoted antinociceptive effect through the activation of descending monoaminergic inhibitory system.

This effect was only seen after systemic administration. However, ketamine absorption from the epidural space to systemic circulation is fast, being impossible to rule out a supra-spinal effect by this route. In rats with peripheral inflammation, ketamine nociceptive effects are clearly independent of the monoaminergic system and are due to anti-NMDA effect. In this situation, there is antinociception regardless of the administration route ${ }^{21}$.

Racemic ketamine is not recommended for epidural and subarachnoid administration due to the neurotoxic potential of the preservative used in its commercial preparation ${ }^{22}$.

$\mathrm{S}(+)$ ketamine is produced in preservative-free solution and has been shown to be superior to the racemic mixture in several ways. Is has four times more affinity for NMDA receptors as compared to $\mathrm{R}(-)$ and higher analgesic potency than the racemic mixture ${ }^{23.24}$. Fewer side effects, such as agitation and psychotic reactions have also been described ${ }^{6}$.

There are some studies on epidural $\mathrm{S}(+)$ ketamine and its subarachnoid administration in continuous infusion for 24 hours has been recently described in chronic pain patient without neurological injury 25-27.

Our study has used an epidural dose of $30 \mathrm{mg}$, considered sub-anesthetic, but results indicated that ketamine through this route has not been effective to control postoperative pain, because numeric and verbal scale scores, time for first analgesic request and postoperative analgesic consumption for both pre and post-incision groups were not significantly different. 
Why was epidural ketamine not effective in this study? Several answers may be proposed. First, NMDA receptors have important role in processing pain perception in the spinal segment. Particularly, these receptors prolong and amplify nociceptive response. All patients received epidural block with local anesthetics before or during surgery, which may have interfered with the action of epidural ketamine on NMDA receptors. Ketamine induces non-competitive NMDA receptor blockade, which means that receptor's channel has to be opened before the drug is bound to it. Epidural blocks with pre-synaptic inhibition of afferent nociceptive receptors could have prevented NMDA receptor of remaining open ${ }^{3}$. Second, and as suggested by some authors, the role of NMDA antagonists on surgical incision-induced pain could be less important than in other experimental pain models. Authors have investigated the effects of NMDA receptor antagonists on mechanical hyperalgesia in a postoperative pain model and have failed to show significant spinal effect ${ }^{28}$. Third, there is a more central site for ketamine anti-hyperalgesic action, which is easily reached after systemic administration. Intraperitoneal surgery also activates afferent nociceptive stimulations (sensory vagus nerve branches) and, finally, ketamine directly interferes with inflammatory reaction. Authors have shown in vitro a potent inhibitory effect of ketamine on different pro-inflammatory cytokines ${ }^{29}$. Systemic ketamine is probably more effective than epidural ketamine for the two latter situations.

There are limited data to determine the preemptive effect of epidural ketamine. Schmid et al. ${ }^{17}$ have observed promising ketamine effects through several routes, including epidural route. Choe et al. ${ }^{30}$, have obtained preemptive effect with epidural ketamine plus morphine in patients submitted to gastrectomy, the same being true for Wong et al. ${ }^{31}$ in patients submitted to knee replacement.

Moiniche, Heklet, Dahl have performed a systematic review of 80 studies on preemptive analgesia ${ }^{32}$, including NSAIDs, intravenous opioids, parenteral NMDA receptor antagonists (intravenous and muscular), epidural analgesia (single dose or continuous infusion), caudal analgesia and local anesthesia. Intravenous ketamine studies were uniformly negative. Only two studies with dextrometorfane, another NMDA antagonist, were effective in promoting preemptive effects.

However, it is worth highlighting that there were only six ketamine studies and it seems more adequate to state that results are still inconclusive.

Only two studies on epidural ketamine alone or in association were included, and it is impossible to conclude that the drug has no preemptive effect through this route.

Pain treatment with continuous epidural injection to determine a preemptive effect extending to the postoperative period has also been surveyed. In theory, this treatment may improve the ability to decrease nociceptive impulses and central neuroplasticity, which are caused not only by surgical incision and procedure but also by postoperative inflammation. However, results have shown that the preemptive effect has been systematically negative, with the proviso that these were only eight studies. Pain intensity improvement has been observed in a small number of cases. An explanation for negative continuous epidural findings may be that notwithstanding the continuous treatment, technique has not been sufficient to prevent triggering and maintenance of central sensitization-induced injury.

It is widely accepted that preemptive analgesia may decrease the risk of postoperative chronic pain. In an attempt to compare the effects of identical pre and post-incision treatments in longer pain periods, some authors have observed that the number of patients with chronic pain six month after surgery was significantly decreased ${ }^{32}$. However it is clear that further data and further preemptive treatment attempts comparing patients receiving no treatment during surgeries such as prostatectomies, thoracotomies or amputations are needed.

Our study has used $17 \mathrm{~mL}$ of $0.25 \%$ bupivacaine plus $3 \mathrm{~mL}$ ( 30 $\mathrm{mg}) \mathrm{S}(+)$ ketamine, which is an analgesic solution volume often used epidurally in association to general anesthesia to promote anesthetic stability without cardiovascular changes. However, the dermatome reached by epidural injection has not been evaluated and it is possible that this volume $(20 \mathrm{~mL})$ was insufficient to totally block afferent nociceptive stimulations triggered by surgical procedure. It is important to remind that adequate somatic analgesia does not necessarily translates into visceral analgesia.

Postoperative analgesia was achieved with bolus $5 \mathrm{~mL}$ of $0.25 \%$ bupivacaine plus $50 \mu \mathrm{g}$ fentanyl as from the first pain complaint until 24 postoperative hours. Bolus was administered on demand at minimum 4-hour intervals, but the injection was given immediately after request to prevent as much as possible any delay in analgesic solution administration and pain intensity increase. The amount of additional analgesics was a means to evaluate preemptive analgesia. $\mathrm{Pa}$ tient-controlled analgesia (PCA) has not been used due to high costs and for not being a routine in the hospital where the study was conducted.

Analgesia was supplemented with $30 \mathrm{mg} \cdot \mathrm{kg}^{-1}$ dipyrone in patients requesting analgesics before the interval of 4-hours. Dipyrone has excellent analgesic action and is not associated to postoperative bleeding because it does not significantly change thromboplastin time or platelet aggregation with the advantage of having spasmolytic action. It decreases postoperative morphine consumption and promotes adequate analgesia. Additionally, there are evidences that dipyrone has a role in central and peripheral sensitization inhibition ${ }^{33-36}$.

It should be highlighted that pain scores in all moments were low, providing high quality analgesia with the proposed technique.

Intercurrences with the studied analgesic approach and the anesthetic technique were mild not leading to patients' dropout.

No studies were found in the literature comparing pre and post-incision administration of epidural $\mathrm{S}(+)$ ketamine plus bupivacaine. Our study has investigated for the first time analgesia obtained with the epidural association of $\mathrm{S}(+)$ ketamine and bupivacaine. 
Recently, Kissin ${ }^{37}$ has reported that the ideal preemptive analgesic approach should avoid the establishment of central sensitization by surgical incision and by the inflammatory process in the early postoperative period. Maybe neuronal plasticity induced during this period is the major reason why several comparative pre and post-incision studies have failed in evaluating preemptive analgesia value.

This model is good enough to assure the presence or absence of the effect, but not to measure its clinical value. Impulses able to start post-surgical hypersensitization may be generated in the pre-incision group, thus decreasing the difference between groups. Still, time for analgesic administration between groups results in higher plasma concentrations of these drugs in the post-incision group, which may have more pronounced effects in preventing hypersensitization in this group and, as a consequence, could decrease the difference between results.

A major aspect also to be reviewed by preemptive analgesia studies is the lack of verification of the pharmacologic effectiveness of the treatment, through direct measurement of the early nociceptive response between preemptive and control groups, measured for example, by the levels of plasma cortisol, $\beta$-endorphins, interleukins, etc. A study in this field with patients submitted to abdominal hysterectomy with epidural bupivacaine and fentanyl has found that analgesic solutions used before surgical incision have not significantly changed serum interleukin- 6 levels, which is a major mediator of the acute phase of inflammatory reaction and responsible for several endocrine and metabolic changes observed in response to surgical trauma ${ }^{38}$.

In conclusion, although having obtained negative results with a combined technique (epidural bupivacaine + $\mathrm{S}(+)$ ketamine), it is sure that further studies should be conducted and that new associations should be tested, including other NMDA receptor antagonists in a longer study, as from before surgical incision until the immediate postoperative period, in an attempt to obtain attenuation of responses which prolong and worsen pain, with wider analgesic coverage throughout this period.

\section{REFERÊNCIAS - REFERENCES}

01. Woolf CJ, Wall PD - Morphine-sensitive and morphine-insensitive action of $\mathrm{C}$-fibre input on the rat spinal cord. Neurosci Lett, 1986;64:221-225.

02. McQuay HJ, Dickenson AH - Implications of nervous system plasticity for pain management. Anaesthesia, 1990;45:101-102.

03. Dickenson AH - Spinal cord pharmacology of pain. Br J Anaesth, 1995;75:193-200.

04. Lodge D, Johnson KM - Noncompetitive excitatory amino acid antagonists. Trends Pharmacol Sci, 1990;11:81-86.

05. Pekoe GM, Smith DJ - The involvement of opiate and monoaminergic neuronal systems in the analgesia effects of ketamine. Pain, 1982;12:57-73.

06. Calvey TN - Isomerism and anaesthetic drugs. Acta Anesthesiol Scand, 1995;106:83-90.
07. Kehlet H - Controlling Acute Pain-Role of Preemptive Analgesia, Peripherical Treatment, and Balanced Analgesia, and Effects on Outcome, em: Max M - Pain: an Updated Review. Seattle, IASP Press, 1999;459-462.

08. McQuay HJ - Preemptive analgesia: a systematic review of clinical studies. Ann Med, 1995;27:249-256.

09. Niv D, Lang E, Devor M - The effect of preemptive analgesia on subacute postoperative pain. Minerva Anestesiol, 1999;65:127-141.

10. Goto T, Marota JJ, Crosby G - Pentobarbitone, but not propofol, produces pre-emptive analgesia in the rat formalin model. $\mathrm{Br}$. J. Anaesth, 1994;72:662-667.

11. Gilron I, Coderre TJ - Preemptive analgesic effects of steroid anesthesia with alphaxalone in the rat formalin test. Evidence for differential $\mathrm{GABA}_{\mathrm{A}}$ Receptor modulation in persistent nociception. Anesthesiology, 1996;84:572-579.

12. Abram SE, Yaksh TL - Morphine, but not inhalation anesthesia, blocks post-injury facilitation. The role of preemptive suppression of afferent transmission. Anesthesiology, 1993;78:713-721.

13. Katz J, Kavanagh BP, Sandler AN et al - Preemptive analgesia. Clinical evidence of neuroplasticity contributing to postoperative pain. Anesthesiology, 1992;77:439-446.

14. Coderre TJ, Katz J, Vaccarino AL et al - Contribution of central neuroplasticity to pathological pain: review of clinical and experimental evidence. Pain, 1993;52:259-285.

15. Kissin I - Preemptive analgesia. Why its effect is not always obvious. Anesthesiology, 1996;84:1015-1019.

16. Amantea B, Gemelli A, Migliorini F et al - Preemptive analgesia o balanced periemptive analgesia? Minerva Anestesiol, 1999;65:19-37.

17. Schmid RL, Sandler AN, Katz J - Use and efficacy of low-dose ketamine in the management of acute postoperative pain: a review of current techniques and outcomes. Pain, 1999;82:111-125.

18. Smith DJ, Pekoe GM, Martin LL et al - The interaction of ketamine with the opiate receptor. Life Sci, 1980;26:789-795.

19. Hustveit O, Maurset A, Oye I - Interaction of the chiral forms of ketamine with opioid, phencyclidine, sigma and muscarinic receptors. Pharmacol Toxicol, 1995;77:355-359.

20. Tung AS, Yaksh TL - Analgesic effect of intrathecal ketamine in the rat. Reg Anesth Pain Med, 1981;6:91-94.

21. Kawamata T, Omote K, Sonoda H et al - Analgesic mechanisms of ketamine in the presence and absence of peripheral inflammation. Anesthesiology, 2000;93:520-528.

22. Malinovsky JM, Lepage JY, Cozian A et al - Is ketamine or its preservative responsible for neurotoxicity in the rabbit? Anesthesiology, 1993;78:109-115.

23. Oye I, Paulsen O, Maurset A - Effects of ketamine on sensory perception: evidence for a role of $\mathrm{N}$-methil-D-aspartate receptors. J Pharmacol Exp Ther, 1992;260:1209-1213.

24. Kohrs R, Durieux ME - Ketamine: teaching an old drug new tricks. Anesth Analg, 1998;87:1186-1193.

25. Koining $\mathrm{H}$, Marhofer $\mathrm{P}$, Krenn $\mathrm{CG}$ et al - Analgesic effects of caudal and intramuscular $\mathrm{S}(+)$ ketamine in children. Anesthesiology, 2000;93:976-980.

26. Himmelseher S, Ziegler-Pithamitsis D, Argiriadou $\mathrm{H}$ et al Small-dose $\mathrm{S}(+)$ ketamine reduces postoperative pain when applied with ropivacaine in epidural anesthesia for total knee arthroplasty. Anesth Analg, 2001;92:1290-1295.

27. Sator-Katzenschlager S, Deusch E, Maier P et al - The long-term antinociceptive effect of intrathecal $S(+)$-ketamine in a patient with established morphine tolerance. Anesth Analg, 2001;93:1032-1034. 
28. Zahn PK, Brennan TJ - Lack of effect of intrathecally administered $\mathrm{N}$-methyl-D-aspartate receptor antagonists in a rat model for postoperative pain. Anesthesiology, 1998;88:143-156.

29. Hill GE, Anderson JL, Lyden ER - Ketamine inhibitis the proinflammatory cytokine-induced reduction of cardiac intracellular cAMP accumulation. Anesth Analg, 1998;87:1015-1019.

30. Choe H, Choi YS, Kim YH et al - Epidural morphine plus ketamine for upper abdominal surgery: improved analgesia from preincisional versus postincisional administration. Anesth Analg, 1997;84:560-563.

31. Wong CS, Lu CC, Cherng CH et al - Pre-emptive analgesia with ketamine, morphine and epidural lidocaine prior to total knee replacement. Can J Anaesth, 1997;44:31-37.

32. Moiniche S, Kehlet H, Dahl JB - A qualitative and quantitative sytematic review of preemptive analgesia for postoperative pain relief: the role of timing of analgesia. Anesthesiology, 2002; $96: 725-741$.

33. Weithmann KU, Alpermann HG - Biochemical and pharmacological effects of dipyrone and its metabolites in model systems related to arachidonic acid cascade. Arzneimittelforschung, 1985;35:947-952.

34. Carlsson $\mathrm{KH}$, Helmereich J, Jurna I - Activation of inhibition from the periaqueductal grey matter mediates central analgesic effect of metamizol (dipyrone). Pain, 1987;27:373-390.

35. Volz M, Kellner HM - Kinetics and metabolism of pyrazolones (propyphenazone, aminopyrine and dipyrone). $\mathrm{Br} \mathrm{J}$ Clin Pharmacol, 1980;10:(Suppl2):299S-308S

36. Shimada SG, Otterness IG, Stitt J - A study of the mechanism of action of the mild analgesic dipyrone. Agents Actions, 1994;41:188-192.

37. Kissin I - Study design to demonstrate clinical value of preemptive analgesia: is the commonly used approach valid? Reg Anesth Pain Med, 2002;27:242-244.

38. Garcia JBS, Issy AM, Salomão R et al - Preemptive analgesia with epidural bupivacaine plus fentanyl in gynaecological surgery - effects on serum interleukin-6 concentrations. Acute Pain, 2002;4:25-28.

\section{RESUMEN}

Castro FE, Garcia JBS - Analgesia Preemptiva con S(+)Cetamina y Bupivacaína Peridural en Histerectomía Abdominal

JUSTIFICATIVA Y OBJETIVOS: El presente estudio investiga la capacidad del antagonista del receptor NMDA, $S(+)$ cetamina, asociado a la inyección peridural de anestésico local (bupivacaína) en promover analgesia preemptiva en pacientes sometidas a histerectomía total abdominal, administrando esa solución antepasadamente a la incisión.

MÉTODO: Fueron evaluadas 30 pacientes, distribuidas aleatoriamente en dos grupos de igual tamaño y estudiadas prospectivamente de forma encubierta. Inyección peridural e inserción de catéter fueron realizadas entre los interespacios de $L_{1}$ - L2 . En el grupo I (G1, $\left.n=15\right)$, las pacientes recibieron por vía peridural, $17 \mathrm{~mL}$ de bupivacaína a $0,25 \%$ sin vasoconstrictor asociados a $30 \mathrm{mg}$ de $\mathrm{S}(+)$ cetamina $(3 \mathrm{~mL})$ treinta minutos antes de la incisión quirúrgica y, después de 30 minutos de la incisión, recibieron $20 \mathrm{~mL}$ de solución fisiológica a $0,9 \%$. En el grupo 2 (G2, $n=15)$, recibieron $20 \mathrm{~mL}$ de solución fisiológica por vía peridural, 30 minutos antes de la incisión, fue hecha una administración de $17 \mathrm{~mL}$ de bupivacaína a 0,25\% asociadas a $30 \mathrm{mg}$ de $\mathrm{S}(+)$ cetamina $(3 \mathrm{~mL})$, treinta minutos después de la incisión. Después de la inyección peridural, se realizó anestesia general con propofol, pancuronio, $\mathrm{O}_{2}$ e isoflurano. Para analgesia post-operatoria fue usada solución peridural en bolus de fentanil asociada a la bupivacaína, con intervalo mínimo de cuatro horas. La suplementación con dipirona solamente era usada si necesario. Se evaluó la intensidad del dolor a través de escala numérica y verbal (al despertar, 6, 12, 1824 horas después del término de la operación), el tiempo necesario para pedir por la primera vez el analgésico y el consumo total de analgésicos.

RESULTADOS: No hubo diferencia significativa entre los grupos con relación al tiempo para pedir analgésicos por la primera vez, al consumo de analgésicos y a los resultados de dolor por las escalas numérica y verbal.

CONCLUSIONES: No fue posible demostrar efecto preemptivo con la utilización peridural de $S(+)$ cetamina y bupivacaína en las dosis utilizadas en histerectomía abdominal. 